\title{
Impacto del crecimiento industrial en la salud de los habitantes de una ciudad minera del Perú
}

\author{
AUGUSTO RAMÍREZ1, TERESITA LEÓN ${ }^{2}$ \\ ${ }^{1}$ Médico del Trabajo. American College of Occupational and Environmental Medicine. \\ ${ }^{2}$ Enfermera Docente Universitaria. Universidad Nacional de Cajamarca. \\ "El cambio del Ambiente es uno de los acontecimientos más dramáticos en la historia de la humanidad, \\ por que cualquier modificación turba inevitablemente a los seres vivientes"
}

\section{Resumen}

Objetivo: Evaluar el impacto que sobre la salud de los habitantes de una ciudad minera de la sierra norte del Perú pudiera haber tenido su incipiente industrialización. Material y Métodos: Empleamos el método retrospectivo descriptivo para revisar las historias clínicas de los pacientes atendidos en el Hospital Regional de la ciudad durante el periodo 1996 al 2 001; buscamos enfermedades relacionadas a contaminación ambiental. Luego, por regresión logística las comparamos estadísticamente con los indicadores de desarrollo industrial de la ciudad. Usamos el programa SPSS/6,1. Resultados: La comparación muestra significancia estadística entre desarrollo industrial y salud de los habitantes: alta cuando correlaciona número de vehículos con enfermedad respiratoria ( $p=0,0001)$, vehículos con enfermedad dermatológica $(p=0,0003)$ y vehículos con conjuntivitis $(p=0,0001)$. La significancia es menor cuando se compara con producción minera de plomo ( $p=0,03,0,001$ y 0,39 , respectivamente) y con producción minera oro/plata $(p=0,01,0,003$ y 0,0045 , respectivamente). No encontramos significancia al comparar desarrollo industrial con incremento de población ni con producción lechera $(p=0,019,0,04)$. Conclusiones: Existe correlación estadísticamente significativa entre crecimiento industrial de la ciudad e incremento de enfermedades de sus habitantes atribuibles a contaminación ambiental. Se recomienda valorar directamente los indicadores de contaminación ambiental a fin de trabajar en su manejo.

Palabras clave: Salud ambiental; contaminación ambiental; enfermedades ambientales; contaminantes industriales.

\section{Industry growth health impact on a mining Peruvian mountain city inhabitants Abstract}

Objective: To determine incipient industrialisation health impact on the population of a mining Peruvian mountain city. Material and Methods: Retrospective descriptive study that reviewed Regional City Medical Hospital patient's records seen from 1996 through 2001, searching for environmental related illnesses. We used growth indexes and compared them statistically with environmental indexes by logistic regression using the SPSS/6,1. Results: We found significant correlation (95\% confidence index) between industrial growth indexes and health status, such as increase of the number of vehicles and respiratory illnesses $(p=0,0001)$, number of vehicles and dermatological illnesses $(p=0,0003)$, number of vehicles and conjunctivitis $(p=0,0001)$. Significance was less when comparing gold/silver production $(p=0,01,0,003$ and 0,0045 , respectively) with lead mining production ( $p=0.03,0,001$, and 0,39 , respectively). There was no significance when comparing industrial development with dairy production and population increase $(p=0,019,0,04)$. Conclusions: Increase in industrial activity correlates with environmental illnesses. We recommend performing further scientific studies to assess environmental pollution index to allow the city to properly manage environmental pollution.

Key words: Enviromental health; enviromental pollution; enviromental illness; industrial pollutants.

Correspondencia:

Dr. Augusto V. Ramírez

Las Mandarinas 210 Ap. 306. Lima 12, Perú

E-mail: augustovram@yahoo.es 


\section{INTRODUCCIÓN}

La contaminación ambiental, aquel fenómeno por el cual la tierra está sufriendo deterioro progresivo, se inicia prácticamente con la era industrial y se agrava luego de la segunda guerra mundial con el auge de la tecnología. Recién a partir de la década de los 70 el hombre comienza a tomar conciencia de la gravedad de este progresivo e irreparable daño. La ecología y ciencias afines se desarrollan y por doquier se implementa acciones para manejar o paliar el daño. La contaminación puede alterar directamente los componentes de un ecosistema, agua, aire o suelos, pero también puede darse por presencia de fenómenos físicos o físicoquímicos inusuales, como calor o radiación ionizante. Sus efectos se manifiestan principalmente por daño a los ecosistemas y por tanto daño a la salud de hombres, animales, vegetales, la tierra o el agua $\left(^{1,2}\right)$.

Se denomina enfermedad ambiental a la producida por exposición a agentes contaminantes presentes en el medio ambiente y que, a diferencia de lo que sucede con otras enfermedades, en ésta no existe primariamente predisposición genética del individuo. Generalmente, el término "enfermedad ambiental" se restringe a enfermedades no infecciosas causadas en su mayor parte por exposición a sustancias presentes en el ambiente, independiente del control inmediato que el enfermo haga sobre su entorno y a la restricción o eliminación de sus hábitos nocivos como fumar o uso de medicamentos, drogas o alcohol. Así, la enfermedad ambiental resulta en una categoría importante de patología referida a una colectividad como resultado de exposición a determinados contaminantes ubicuos en el medio $\left(^{3}\right)$.

Contaminación ambiental es definida como "todo cambio indeseable causado por el hombre en las características del aire, agua o suelo que afectan negativamente a los seres vivientes y en general al ecosistema". El problema, como hemos visto, comienza con la revolución industrial a finales del siglo XVIII con el invento de la máquina a vapor y su hija inmediata, la máquina de tejer. Hasta entonces, la producción humana se hacía artesanalmente y la comercialización de los productos tenía cuasi un carácter de trueque. Se inicia así la gran etapa de la fábrica como sistema de producción masiva lo que trae avance tecnológico y desarrollo tan preciados hoy en día. Sin embargo, a resultas de lo mismo y concomitantemente otro proceso sigiloso y paulatino comienza y nos está llevando la destrucción de nuestro hábitat natural. En los años 40 del siglo pasado, se produce otra onda tecnológica, que algunos han considerado radical, la llaman "industria de guerra" y por supuesto en esos momentos graves a nadie le importa el deterioro del ambiente $\left(^{1-4}\right)$.

Pero, por qué se deteriora la atmósfera? Entre otras causas, la atmósfera se deteriora por acumulación de gases llamados gases de efecto invernadero (GEI), resultantes del uso de combustibles fósiles, incremento desordenado de las industrias, expansión de la ganadería y deforestación galopante. De otro lado los cambios climáticos aumentan los episodios de contaminación atmosférica por incremento de los movimientos circulantes en la atmósfera y la consiguiente dispersión de los contaminantes. Los GEI más importantes son dióxido de carbono, oxido nitroso, metano y los fluoroclorocarbonos. Los GEI afectan la salud humana de dos maneras, primero indirectamente con el aumento de temperatura, humedad y lluvias en una determinada región, lo que favorece el desarrollo de enfermedades metaxénicas cuyos vectores dependen de la variación climática; segundo, directamente, pues conllevan aumento de enfermedades relacionadas de manera directa a la contaminación. Adicionalmente, la contaminación disminuye la capa de ozono e incrementa la radiación UV con aumento de lesiones dérmicas y oculares $\left(^{5-7}\right)$.

En el recuento histórico, el concepto de enfermedad ambiental tuvo su origen en el reconocimiento de enfermedad ocupacional, quizá porque es más fácil correlacionar exposiciones intensas y sostenidas con determinada entidad nosológica, p. ej., neumoconiosis en exposición a polvo de sílice libre o cáncer de escroto en 
limpiadores de chimeneas expuestos a hollín. Muchas de estas enfermedades llamaron ya la atención en el inicio de la revolución industrial en el siglo XVIII. Hoy son harto conocidos los desastres ecológicos de Londres, Donora, Minamata, Chernobyl, Bophal, del Golfo Pérsico, del Danubio originado en Baia Mare o los de petróleo en los mares $\left({ }^{8}\right)$.

En el Perú, sabemos del daño ambiental de la Ciudad de la Oroya, del lago de Junín, el derrame de Choropampa y la contaminación urbana por polvo de plomo a partir de los depósitos de concentrados de minerales en el Callao. Además, en el interior, las ciudades pequeñas han comenzado a sentir el impacto del deterioro de su medio ambiente, pues de villas y villorrios pasan bruscamente a ciudades de industrialización emergente. Desde hace algunas décadas, el aire de la mayoría de las capitales de los países latinoamericanos se ha deteriorado, como consecuencia de la instalación de industrias en su periferia y del rápido crecimiento del obsoleto parque automotor que usa combustible fósil con aditivos de plomo. La migración no planificada del campo a la ciudad contribuye al agravamiento por la tugurización de las ciudades de crecimiento intermedio. El fenómeno mundial de globalización y el desempleo son factores condicionantes que han llevado a la domiciliarización del trabajo $\left({ }^{9}\right)$.

En nuestro país, a resultas de la pobreza, las ciudades medianas y sobre todo las capitales de departamento se ven "invadidas" en forma no planificada por gran cantidad de inmigrantes que dejan el campo y llegan con su grupo familiar buscando sobrevivir. El crecimiento rápido de las ciudades medianas o intermedias tiene este substrato, pues con la migración crecen en forma desordenada y este aumento de población incrementa la necesidad de servicios públicos, como transporte, agua, desagüe, energía. Por otra parte, estos cambios dan lugar a la aparición de micro y pequeñas industrias (fábricas clandestinas de acumuladores eléctricos, soldadores, "reparadores" caseros de electrodomésticos, de piezas de automóviles en la vía pública, comercio ambulatorio, etc.). En suma, la domiciliarización de los riesgos del trabajo. La ciudad de nuestro estudio es un buen ejemplo de esto; desde hace 10 ó 12 años inicia una etapa activa de crecimiento impulsado principalmente por el desarrollo de la actividad minera que trae capitales y puestos de trabajo bien remunerados, pero que a su sombra da lugar al crecimiento no muy ordenado o precario de industrias conexas.

Precisamente, con el presente estudio diseñado como retrospectivo descriptivo y de hipótesis asociativa nos proponemos averiguar hasta qué punto podemos correlacionar crecimiento industrial de la ciudad con deterioro en la salud de sus habitantes $\left({ }^{10-13}\right)$. Estudiamos retrospectivamente el lapso 2001 - 1996 para investigar el comportamiento de diferentes parámetros socioeconómicos como causantes de alteraciones del medio ambiente, tales como crecimiento de población, parque automotor, consumo de energía, aumento de producción minera, lechera y ganadera. Concomitantemente, verificamos en su Hospital Regional del Ministerio de Salud, la variación de las enfermedades atribuibles a contaminación del medio ambiente, principalmente respiratorias, oculares y cutáneas. Por el corto tiempo de desarrollo industrial de la ciudad (10 a 12 años), no investigamos enfermedades degenerativas o mutagénicas que, en el largo plazo, también han sido atribuidas a contaminación ambiental.

\section{MATERIAL Y MÉTODOS}

Los objetivos del estudio fueron verificar el crecimiento de una "nueva" ciudad industrial, verificar si concomitantemente hubo incremento de enfermedades relacionadas con el deterioro del medio ambiente y, de haber aumentado las enfermedades ambientales, investigar si estadísticamente este aumento puede ser atribuido a la modificación medio ambiental causada por la industrialización de la ciudad.

El estudio fue diseñado como retrospectivo y descriptivo de hipótesis asociativa con relación de dependencia. 
El crecimiento industrial lo determinamos averiguando la variación de los indicadores económicos, entre los que destacamos:

- Crecimiento de la población.

- Crecimiento del parque automotor.

- Crecimiento de la producción minera y ganadera.

- Incremento del consumo de energía eléctrica $\left({ }^{14}\right)$.

La población estudiada comprendió al total de la población atendida en el Hospital Regional del Ministerio de Salud, en lapsos anuales. Revisamos las historias clínicas archivadas en la sección Estadística, buscando enfermedades de aceptada etiología ambiental, como reacción irritante primaria local de vías respiratorias, piel y ojos, clasificadas por CIE (Clasificación Internacional de Enfermedades ) 10:

- Reacción irritante primaria local directa en el sistema respiratorio superior: Rinitis, faringitis y traqueitis, J 30,4 a 30,6, J 31,2. Asma bronquial, J 45 y Broncoespasmo, J 98,0.

- Dermatitis no infecciosa, L 23,9.

- Conjuntivitis no bacteriana, H 10,1 ( $\left.{ }^{15}\right)$.

Se realizó el análisis estadístico usando el software SPSS $/ 6,1^{\circledR}$. Para evaluar las diferencias entre las medias usamos la prueba " $t$ " de Student. La correlación entre las variables cuantitativas se valuó por el coeficiente de Pearson, situando la probabilidad $p$ de error crítico a nivel de 0,05. Para identificar factores que contribuyan a explicar la variación de los parámetros estudiados, usamos el análisis de regresión linear múltiple $\left({ }^{16,17}\right)$.

La limitación de nuestro estudio fue no cubrir el total de consultas de la población, pues el lugar de nuestro estudio, Hospital Regional del Ministerio de Salud, atiende aproximadamente sólo a 70\% del total, mientras el resto se atiende en el Hospital de Seguridad Social (EsSalud) y en clínicas particulares.

\section{RESULTADOS}

La descripción de la población estudiada se presenta en la Tabla 1, donde se aprecia las características de la población atendida en el Hospital Regional y los de la muestra. La media de edad de la población atendida en el Hospital fue 31,7 años, de la muestra 24,8 años, de ésta $54,4 \%$ fueron mujeres y $45,6 \%$ varones. Es claro que la mayor parte de atendidos fueron menores. No encontramos diferencia significativa entre sexos.

Tabla 1.- Características de la población estudiada.

\begin{tabular}{lcc}
\hline \multicolumn{1}{c}{ Tipo } & $\begin{array}{c}\text { Población } \\
\text { atendida }\end{array}$ & $\begin{array}{c}\text { Población } \\
\text { estudiada }\end{array}$ \\
\hline Media de edad (años) & 31,7 & 24,8 \\
Sexo femenino (\%) & 54,4 & 51,0 \\
Sexo masculino (\%) & 45,6 & 49,0 \\
\hline
\end{tabular}

Fuente: Departamento de Archivo y Estadística Hospital Regional de la ciudad estudiada. Ministerio de Salud. Perú, 2002.

En la Tabla 2 se aprecia que en el lapso estudiado hubo aumento sostenido de la actividad industrial: el parque automotor pasó de 5006 vehículos a 8 893, el consumo de energía de 5 984978 a $9519152 \mathrm{kw} / \mathrm{h}$, el incremento de la población de 153345 a 174269 habitantes, la producción de leche de 84504 litros a 178306 litros, el número de cabezas de ganado se incrementó de 861794 a 2105 442, la producción de oro/plata de 111415 a 164796 kilos y la de plomo de 4040 a 7958 toneladas.

La Tabla 3 muestra las enfermedades de fondo alérgico distribuidas por año y atendidas en el Hospital Regional en el periodo 1996 a 2001. Vemos un incremento sostenido largo del periodo. Así, las dermatitis aumentaron de 802 a 2 309, las respiratorias totales de 962 a 3411 y las irritativas oculares de 11 a 310 . Es decir, en ese lapso, las dermatitis se incrementaron 3 veces, las respiratorias 3,5 veces y las conjuntivitis en mucho mayor cantidad, aún cuando su incidencia relativa en el universo estudiado fue pequeña.

En la Tabla 4 encontramos la comparación estadística del incremento de enfermedad 
Tabla 2.- Crecimiento industrial de la ciudad, $1996-2001$.

\begin{tabular}{lrrrrrr}
\hline Tipo actividad industrial/ año & 1996 & 1997 & 1998 & 1999 & 2000 & 2001 \\
\hline Vehículos, n & 5006 & 8006 & 8414 & 8648 & 8771 & A 8 893 \\
Consumo energía kw/h & 5984978 & 7769817 & 9714972 & 7996390 & 9296272 & 9519152 \\
Población b n & 153345 & 157379 & 161530 & 165728 & 170003 & 174269 \\
Producción minera plomo. Ton & 4040 & 5445 & 7190 & 8993 & 7586 & 7958 \\
Producción minera oro/plata. K & 111415 & 117755 & 150338 & 171213 & 193315 & 164796 \\
Producción lechera. L. & 84504 & 114538 & 119658 & 145655 & 153603 & 178306 \\
$N^{\circ}$ cabezas de ganado, n & 861794 & 1049064 & 2143206 & 2107070 & 1817096 & 2105442 \\
\hline
\end{tabular}

a Estimados a noviembre 2001.

b Estimada según Censo Nacional 1993.

Fuente: Instituto Nacional de Estadística e Informática. Perú. Almanaque Estadístico, 2002.

Tabla 3.- Total enfermedades ambientales por año.

\begin{tabular}{lcrrr}
\hline Año & $\begin{array}{c}\text { Total } \\
\text { dermatitis }\end{array}$ & $\begin{array}{c}\text { Total } \\
\text { respiratorias }\end{array}$ & Conjuntivitis & Total \\
\hline 1996 & 802 & 962 & 11 & 1775 \\
1997 & 1527 & 1414 & 22 & 2963 \\
1998 & 1589 & 2399 & 95 & 4083 \\
1999 & 1695 & 3479 & 112 & 5286 \\
2000 & 1600 & 3185 & 221 & 5006 \\
2001 & 2309 & 3411 & 310 & 6030
\end{tabular}

Fuente: Departamento de Archivo y Estadística Hospital Regional de la ciudad estudiada Ministerio de Salud. Perú, 2002. alérgica atribuible al aumento de la actividad industrial, los valores del ratio de la diferencia (RD), el intervalo de confianza (IC) al $95 \%$ y el valor "p" de Pearson. Al análisis se aprecia que estadísticamente el incremento de enfermedades ambientales estuvo directamente ligado al crecimiento industrial.

Las Figuras 1 y 2 muestran respectivamente el aumento de enfermedades ambientales y el incremento de la actividad industrial entre los años 1996 y 2001.

Tabla 4.- Comparación estadística de enfermedad ambiental vs actividad industrial.

\begin{tabular}{|c|c|c|c|c|c|c|c|c|c|c|c|}
\hline \multirow{2}{*}{$\begin{array}{l}\text { Enfermedad/ } \\
\text { actividad industrial } \\
\text { Tipo }\end{array}$} & \multicolumn{2}{|c|}{$\begin{array}{l}\text { Valores inicio - fin } \\
\text { del periodo medido }\end{array}$} & \multicolumn{3}{|c|}{$\begin{array}{l}\text { Enfermedad } \\
\text { respiratoria alérgica a }\end{array}$} & \multicolumn{3}{|c|}{ Dermatitis alérgica } & \multicolumn{3}{|c|}{$\begin{array}{c}\text { Conjuntitis } \\
\text { no bacteriana }\end{array}$} \\
\hline & 1996 & 2001 & $\mathrm{RD}$ & IC $95 \%$ & $\mathrm{p}^{\mathrm{b}}$ & $\mathrm{RD}$ & IC $95 \%$ & $\mathrm{p}^{\mathrm{b}}$ & $\mathrm{RD}$ & IC $95 \%$ & $\mathrm{p}^{\mathrm{b}}$ \\
\hline Número vehículos, n & 5006 & c8 893 & 1,0 & $0,5-2,2$ & 0,0001 & 1,2 & $0,5-3,1$ & 0,0003 & 0,8 & $0,6-1,1$ & 0,001 \\
\hline Consumo energía $\mathrm{kw} / \mathrm{h}$ & 5984978 & 9519152 & 1,2 & $0,5-8,1$ & 0,01 & 1,2 & $0,8-1,9$ & 0,13 & 0,9 & $0,3-2,1$ & 0,027 \\
\hline Población n & 153345 & 174269 & 1,2 & $0,5-2,9$ & 0,04 & 1,1 & $0,6-2,1$ & 0,04 & 4,9 & $2,5-9,7$ & 0,05 \\
\hline Producción plomo. Ton & 4040 & 7958 & 1,1 & $0,6-2,0$ & 0,03 & 2,0 & $1,1-3,7$ & 0,001 & 4,9 & $2,5-9,7$ & 0,039 \\
\hline Producción oro/plata. K & 111415 & 164796 & 1,1 & $0,5-2,0$ & 0,01 & 1,9 & $1,3-3,2$ & 0,003 & 4,9 & $2,0-8,1$ & 0,045 \\
\hline Producción lechera 1. & 84504 & 178306 & 0,9 & $0,3-1,0$ & 0,019 & 0,9 & $0,6-1,4$ & 0,05 & 4,7 & $1,6-14$ & 0,361 \\
\hline Ganadería total, $\mathrm{n}$ & 861794 & 2105442 & 1,0 & $0,2-1,1$ & 0,01 & 1,0 & $0,6-1,5$ & 0,04 & 4,8 & $2,1-9,7$ & 0,032 \\
\hline
\end{tabular}

$R D=$ ratio de diferencias (odds ratio)

$I C=$ intervalos de confianza

a Incluye Rinitis, Faringotraqueitis, Asma bronquial y Broncoespasmo.

${ }^{b} p=$ valor $p$ de Pearson

${ }^{c}$ Estimado a Nov. 2001 


\section{DISCUSIÓN}

La valoración de los efectos de la contaminación ambiental sobre la salud se hace desde dos puntos de vista, uno directo que consiste en medir los contaminantes del ambiente y referirlos a cifras preestablecidas que se considera "seguras", y el otro indirecto, por el cual se verifica el deterioro de la salud de los habitantes de un determinado ecosistema, hombres, animales o plantas $\left({ }^{18}\right)$.

En la medición del impacto en salud es importante valorar los efectos agudos o sub agudos que producen en las poblaciones expuestas, pues todos condicionan o agravan enfermedades crónicas respiratorias, cutáneas u oculares. Por ejemplo, el ozono y los $\mathrm{NO}_{\mathrm{x}}$ (oxidos nitrosos), gases ubicuos en una ciudad, producen aumento de síntomas respiratorios y disminución de la función pulmonar; mercurio, cromo, níquel, HCN (cianuros), alquitrán, benzopirenos o arsénico producen lesiones en piel y ojos; material particulado unido a $\mathrm{SO}_{\mathrm{x}}$ (oxidos sulfurosos) aumenta la frecuencia y morbilidad de las enfermedades respiratorias estacionales y alérgicas, desencadenan ataques de asma, aumentan la morbilidad en los portadores de enfermedad pulmonar obstructiva crónica (EPOC); el monóxido de carbono disminuye la capacidad de ejercicio físico en adultos saludables y aumenta la morbilidad de los portadores de enfermedad cardiaca isquémica. El problema se agrava porque actúan sinérgicamente y existe relación directa entre enfermedades crónicas o degenerativas (cáncer, embriogénicas, mutagénicas, etc.) e industrialización-contaminación por emisiones ambientales "de rutina" (plomo, asbesto, dioxinas, policloruros de bifenilo); por las fugas accidentales $\left(\mathrm{H}_{2} \mathrm{SO}_{4}\right.$, etilenglicol, mercurio, amonio, petróleo crudo o refinado, $\mathrm{HCN}$ ) o por mala disposición de desechos industriales tóxicos (arsénico, níquel, plomo, cromo, cadmio) $\left({ }^{16,20,21}\right)$, pero por la naturaleza crónica de este tipo de exposición ambiental sus efectos tardan años en manifestarse $\left({ }^{19-22}\right)$.

En el Perú existen investigaciones puntuales sobre el efecto dañino para la salud de nuestros habitantes, principalmente en ciudades industriales y en ciudades aledañas a instalaciones minero metalúrgicas. Sin embargo, a la fecha no tenemos un consolidado o inventario de la evolución de estas enfermedades, visto que en los últimos años ciudades de tamaño pequeño o intermedio han comenzado a desarrollarse en forma desordenada con un mosaico de micro o pequeñas industrias, incluyendo industrias "caseras", cuyos trabajadores pertenecen al mismo grupo familiar del dueño, y se puede afirmar en el 100\% de los casos, estos trabajadores desconocen los riesgos que enfrentan y generan $\left.{ }^{(23-25}\right)$.

Precisamente nuestro estudio se orienta a valorar el impacto producido por el crecimiento industrial en los habitantes de la ciudad estudiada, contrastándolo con aumento de enfermedades atribuibles al deterioro ambiental urbano causado por su crecimiento. Para evaluarlo seleccionamos parámetros aceptados internacionalmente como indicadores de desarrollo industrial $\left({ }^{26}\right)$.

El análisis de los resultados de nuestro estudio mostró incremento sostenido de las enfermedades ambientales paralelo al desarrollo industrial. La enfermedad respiratoria presentó su mayor significancia estadística al compararla con aumento de número de vehículos valor de $p=$ a0,0001, lo que concuerda con los estudios de Ramírez-Bejar y Andrade en Ilo, Perú $\left({ }^{27,28}\right)$ y con los de Nowak en Alemania $\left({ }^{29}\right)$, Ginn en el Reino Unido $\left({ }^{30}\right)$, Keidin en Holanda $\left({ }^{31}\right)$ y con la American Thoracic Society $\left({ }^{32}\right)$. Hallamos también correlación significativa pero de menor cuantía cuando la correlacionamos con producción lechera $p=0,019$, consumo de energía $p=0,01$, con ganadería $p=0,01$ y con producción minera de plomo y de plata/oro $p=0,01$ ambos. Pero, la correlación no fue significativa al compararla con aumento de población, $p=0,05$.

Al contrastar el incremento de dermatitis, también obtuvimos alta correlación con aumento del número de vehículos $p<0,0003$, con producción minera de plomo $p=0,001$ y con la de oro/plata $p=0,003$. En menor grado se correlacionó con producción ganadera $p=0,039$ 


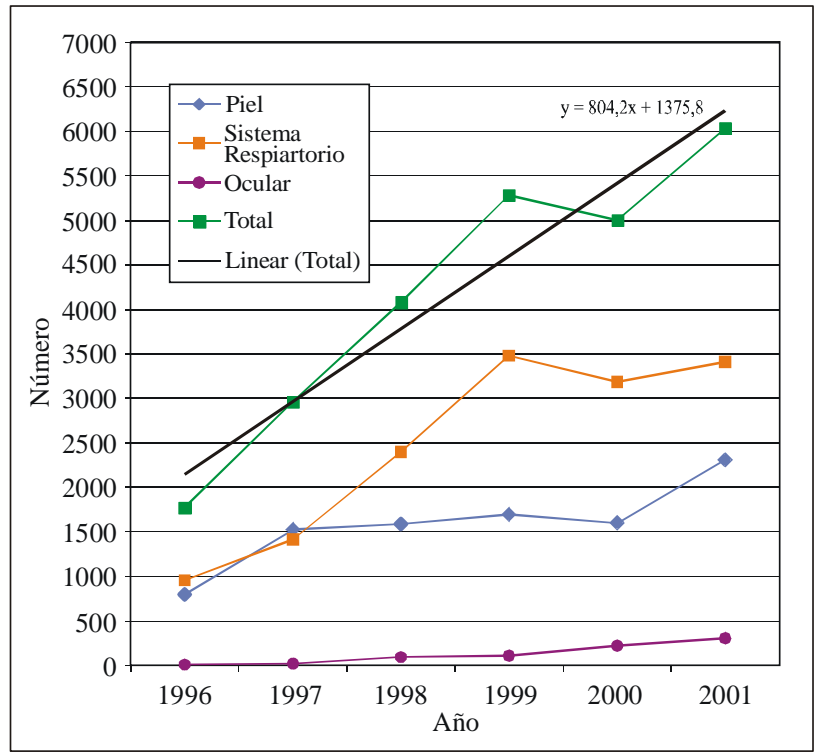

Figura 1.- Incremento enfermedad ambiental por año, 1996-2001.

y producción lechera $p=0,04$. Finalmente, la correlación no fue significativa cuando comparamos dermatitis con aumento de población $p=0,06$ ni con aumento de consumo de energía $p=0,13$. Excepto la variable aumento de población, las otras concuerdan con el análisis Brack y León en Perú $\left({ }^{10,33}\right)$ y con Katsouyanni en Europa $\left({ }^{34}\right)$ además de lo sostenido en medicina e higiene ambiental $\left({ }^{20,22,35}\right)$.

La correlación para conjuntivitis y actividad industrial fue positiva en las variables estudiadas, tal como lo describen Rom, Aunam e Isselbachen $\left({ }^{18,36,37}\right)$. Sin embargo, para producción de leche el valor de $p$ es 0,36 y para población 0,05 , por lo que en estos dos últimos casos nuestra correlación no fue significativa.

Creemos que la asociación con aumento de población no se correlaciona con las variables investigadas, porque el número de habitantes indicado por el Instituto Nacional de Estadística e Informática (INEI) es sólo una proyección estadística a partir del censo de 1993; desde entonces, no hemos tenido un censo nacional, y por tanto no toma en cuenta la fuerte inmigración

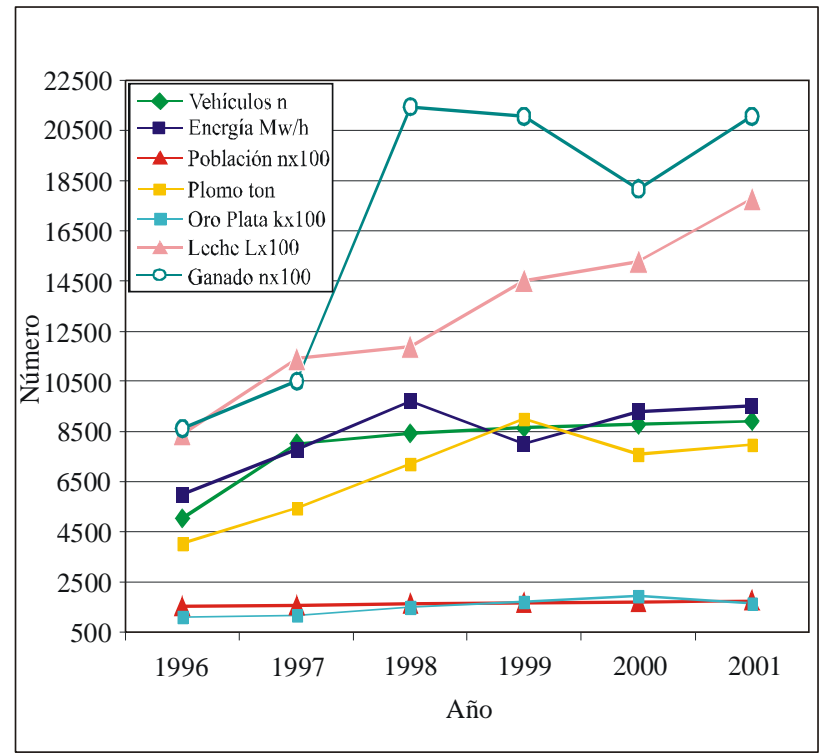

Figura 2.- Crecimiento industrial de la ciudad, 1996-2001.

recibida por la ciudad debida al auge de la actividad minera del oro, que inicia la ciudad precisamente en $1993\left({ }^{14}\right)$.

Por otro lado, la disminución puntual de la actividad industrial ocurrida el año 2000, creemos fue ocasionada indirectamente como una respuesta a un conocido derrame accidental de mercurio en sus cercanías. A pesar de esta disminución, las consultas por enfermedad ambiental mantienen su tendencia y al año siguiente recobran su ascenso, lo que es explicable porque ese mismo evento determinó incertidumbre en la población.

Por los resultados de nuestro estudio, concluimos que el temprano desarrollo industrial de la ciudad estudiada ha traído también un aumento estadísticamente significativo de las enfermedades relacionadas a contaminación ambiental, principalmente respiratoria, dérmica y ocular, en ese orden.

Para consolidar estos resultados recomendamos investigar directamente variables de contaminación ambiental, como plomo sanguíneo en la población, plomo, metales 
pesados, material particulado, $\mathrm{CO}$ (monóxido de carbono), $\mathrm{SO}_{\mathrm{x}}, \mathrm{NO}_{\mathrm{x}} \mathrm{y}$ otros en el ambiente.

\section{REFERENCIAS BIBLIOGRÁFICAS}

1. Nebel BJ. Ciencias Ambientales. Ecología y Desarrollo sostenible. 6 ${ }^{a}$ Ed. México: Prentice Hall; 1999.

2. Clark W de P Jr. Occupational and Environmental Diseases. Encyclopædia. New York: Microsoft Corporation; 2003.

3. La Dou J, Jackson RT, Howard JJ. Salud ambiental. En: La Dou J. Medicina Laboral y Ambiental. $2^{\mathrm{a}}$ Ed. México: Ed. Manual Moderno; 1999.

4. Jeyaratnam J. Transfer of hazardous industries. En: Occupational Cancer in Development Countries. Lyon: IARC; 2000.

5. Samel JM. Community Air Pollution. En: Ron WN (Ed). Environmental Medicine $3^{\text {a }}$ Ed. USA: Lippincott-Raven; 1997.

6. Rubin F. Patología: Gases Irritantes. $4^{\mathrm{a}}$ Ed. Buenos Aires: Ed Panamericana; 1994.

7. Deulin R. Air Pollutants: Human Health Studies. En: Ron WN (Ed). Environmental Medicine $3^{\text {a }}$ Ed. USA: LippincottRaven; 1997.

8. Baxter PJ. Major chemical disasters. Br Med J 1991;302:61.

9. Weitzenfeld DH. Contaminación Atmosférica y Salud en América Latina. Bol Oficina Sanit Panam 1992;112:97-109.

10. Brack EA. Perú y Ambiente, uno de los desafíos del siglo XXI. Asociación Nacional de Centros, IV Conades-PNUD, Lima, julio 2000.

11. Organización Panamericana de la Salud. La Salud y el Ambiente en el desarrollo sostenible. Washington DC: OPS; 2000. Publicación Científica 572.

12. Pastore J. A agonia do Emprego. Sao Paulo, Brasil: Ed. Renacemento; 1998.

13. Mc Michael A J, Ed. Climate Change and Human Health: An assessment prepared by a Task Group on Behalf of the World Health Organization. The World Meteorological Organization and the United Nations. Geneva: WHO; 1996.

14. Instituto Nacional de Estadística e Informática. Almanaque Estadístico. Lima: INEI; 2002.

15. World Health Organization. 1992 International Classification of Diseases and Related Health Problems. Geneva: WHO; 1992.

16. Camacho RJ. Estadística con SPSS para Windows. España: diciones Ra-Ma; 2000.

17. Fleiss JL. Statistical Methods for Rates and Proportions. 2nd Ed USA: Wiley; 1981.

18. Rom WN, Ed. Environmental Medicine. USA: LippincottRaven; 1995.

19. Zenz C, Ed. Occupational Medicine, 3th Edition. St. Louis, USA: Ed Mosby - Year Book Inc.; 1994.
20. Gradel TE, Allenby BR. Industrial Ecology. USA: PrenticeHall; 1995.

21. Doa MJ. The toxic release inventory hazard waste. Hazard Mater 1992;9:61.

22. Ellemhorn MJ. Medical Toxicology. Penn, USA: Williams \& Wilkins; 1997.

23. Ramírez AV, Paucar JC,Medina JM. Plomo sanguíneo en los habitantes de cuatro localidades peruanas. Pan Am J Public Health 1997;1(5):334-48.

24. Ramírez AV. Cadmium pollution in La Oroya, Peru. P A H O Bulletin 1986;20(4):375-9.

25. Low P, Yeats A. Do Dirty Industries Migrate? En: Patrick L (Ed). International Trade an Environment. USA: The World Bank; 1992.

26. Judge GG, Friffiths WE, Hill RC, et al. Theory and Practice of Econometric. New York: Wiley and Sons; 1980

27. Ramírez AV, Bejar K. Espirometría en estudiantes de una ciudad industrial. An Fac Med 2001;62(3).

28. Andrade MP. Asma Bronquial Prevalencia, Severidad y Factores Precipitantes y de Riesgo en escolares de 13-14 años de la Ciudad de Ilo. Tesis de Bachiller en Medicina Humana Universidad Nacional de San Agustín, Arequipa 1997.

29. Nowak D, Heinrich J, Jorres R, et al. Prevalence of respiratory symptoms, bronchial hyper responsiveness and atopy among adults: west and east Germany. Europ Respir J 1996;9(12):2541-52.

30. Ginns SE, Gatrell AC. Respiratory health effects of industrial air pollution: a study in east Lancashire, UK. J Epidemiol Community Health 1996;50(6):631-5.

31. Keiding LM, Rindel AK, Kronborg D. Respiratory illnesses in children and air pollution in Copenhagen. Arch Environ Health 1995;50(3):200-6.

32. American Thoracic Society. Health effects of outdoor air pollution Part 1 and 2. Committee of the Environmental and Occupational Health Assembly of the American Thoracic Society. J Respir Crit Med 1996;153(1-2):3-50.

33. León RMT. Alergias producidas por Factores Ambientales. Tesis de Maestría. Escuela de Pos Grado Universidad Nacional de Cajamarca. Perú. Nov. 2001.

34. Katsouyanni K, Touloumi G, Spix C, et al. Short-term effects of ambient sulfur dioxide and particulate matter on mortality in 12 European cities: results from APHEA project. BMJ 1977;314:1658-63.

35. Somis A. Contaminación Atmosférica. Medicina Sanitaria y Administración de Salud. $2^{\text {a }}$ Edición. Buenos Aires: Ed. El Ateneo; 1982.

36. Aunan K. Exposure-response functions for health effects of air pollutants based on epidemiological findings. Risk Anal 1996;16(5):693-709.

37. Isselbacher KJ. En: Harrison Principios de Medicina Interna. 13a Edición. Madrid: Ed Interamericana Mc Graw Hill; 1994. 